\title{
Local amplification of Rayleigh waves in the continental United States observed on the USArray
}

\author{
Celia L. Eddy ${ }^{\mathrm{a}, *}$, Göran Ekström ${ }^{\mathrm{a}}$ \\ ${ }^{a}$ Department of Earth and Environmental Sciences, Columbia University, 61 Route $9 \mathrm{~W}$, \\ Palisades, NY 10964 USA
}

\begin{abstract}
We develop a method based on ratios of amplitudes measured at adjacent stations to determine local amplification of surface waves across an array of seismic stations. We isolate the effects of local structure from those of the earthquake and propagation by systematic averaging of ratios corresponding to many sources. We apply the method to data recorded on the USArray for the years 2006-2011 and determine amplification factors at each station of the array for Rayleigh waves at periods between $35 \mathrm{~s}$ and $125 \mathrm{~s}$. Local amplification factors are spatially coherent and display variations of $\pm 10 \%$ at a period of $125 \mathrm{~s}$ and greater variations at shorter periods. Maps of local amplification exhibit spatial correlation with topography and geologic structures in the western and central United States. At long periods, the observed amplification factors correlate well with predictions from a regional crust and mantle model of North America. At short periods, correlations are weaker, suggesting that the local amplification factors can be useful for constraining shallow structure better.
\end{abstract}

\footnotetext{
*Corresponding author

Email address: ceddy@ldeo.columbia.edu (Celia L. Eddy)
} 
Keywords: seismology, surface wave, amplitude, USArray

1

3

4

\section{Introduction}

Surface waves provide one of the principal constraints on structure in the uppermost part of the Earth. Both phase and amplitude, the two primary surface-wave observables, contain useful information about structure in the crust and upper mantle. Several different factors contribute to affect the amplitudes of surface waves, including source, path, and receiver effects (e.g., Selby and Woodhouse, 2000; Dalton and Ekström, 2006b). In addition to this complexity, stations are sometimes poorly calibrated in amplitude. Because of this, amplitude data are used less frequently than phase travel time data in tomography studies. In recent decades, significant work has been done using measurements of surface-wave phase travel times to constrain both two-dimensional (e.g., Zhang and Tanimoto, 1991; Trampert and Woodhouse, 1995; Ekström et al., 1997; Trampert and Woodhouse, 2003; Ekström, 2011; Lin and Ritzwoller, 2011) and three-dimensional (e.g., Masters et al., 1996; Boschi and Ekström, 2002; Shapiro and Ritzwoller, 2002; Kustowski et al., 2008; Ritsema et al., 2011) velocity structure in the Earth. Although some studies model both surface-wave phase and amplitude using two-plane and multi-plane wave methods (e.g., Yang and Forsyth, 2006; Pollitz and Snoke, 2010), less work has been done to constrain Earth structure using amplitude data. Surface-wave amplitudes are, however, a potentially rich source of information about both elastic and anelastic structure of the crust and upper mantle.

Surface-wave amplitudes contain information about propagation effects, 
including both attenuation and elastic focusing (Selby and Woodhouse, 2000).

The majority of surface-wave amplitude studies have focused on constraining anelastic structure of the crust and upper mantle. Previous studies that have developed global models of surface-wave attenuation include Durek et al. (1993), Selby and Woodhouse (2002), Gung and Romanowicz (2004), and Dalton and Ekström (2006b). The use of amplitude measurements to infer elastic structure has been less common (e.g., Dalton and Ekström, 2006a).

In addition to propagation effects, recorded surface-wave amplitudes include an amplification factor that depends on the instrument response and Earth structure local to the station. Ideally, the instrument response is known perfectly, and the receiver factor can be attributed entirely to Earth structure. Unfortunately, the absolute instrument gain is difficult to measure and verify, and is therefore associated with significant uncertainty. Errors in the seismometer gain of several percent or more are common. For example, Ekström et al. (2006) investigated the calibration of stations in the Global Seismographic Network and discovered constant and time-dependent calibration errors of the order of $10 \%$ at a significant fraction of the stations. At this level, calibration errors may be the dominant contribution to the receiver factor. Interpretation of receiver-amplitude factors in terms of Earth structure is therefore difficult and depends critically on the quality of the station calibration.

The Earth structure contribution to the receiver-amplitude factor is a local effect that depends on the elastic structure beneath the station (e.g., Wang and Dahlen, 1994; Ferreira and Woodhouse, 2007b). Relatively few studies have investigated this site effect in part because of the difficulties in 
making accurate measurements of surface-wave amplitude. However, some recent work has demonstrated how local elastic structure can be inferred from surface-wave amplitudes. For example, Ferreira and Woodhouse (2007a), Tanimoto and Rivera (2008), Yano et al. (2009), and Lin et al. (2012a) showed that the ratio between vertical and horizontal Rayleigh wave amplitudes can be used to determine radial shear-velocity structure beneath stations. Recently, Lin et al. (2012b) used measurements of surface-wave phase and amplitude recorded on the USArray to derive maps of local amplification across the western United States using Helmholtz tomography. Local surface-wave amplification in the continental United States is also the focus of this paper.

Surface-wave amplitudes can provide a complementary constraint to phase velocity on elastic structure in the crust and upper mantle. Sensitivity of amplification and phase velocity to perturbations in $v_{P}, v_{S}$, and density, $\rho$, is shown in the radial sensitivity kernels in Figure 1. The phase velocity kernels for velocity perturbations are always positive, while the amplification kernels change sign with depth. Peak sensitivity of surface-wave amplification to velocity perturbations is slightly shallower than the peak of phase velocity sensitivity. In addition, surface-wave amplitudes are more sensitive than phase velocity to shallow crustal structure. Although the density sensitivity is relatively small compared to the velocity sensitivity, amplification is more sensitive than phase velocity to density perturbations. Because of these different sensitivities, combining observations of surface-wave amplitude with measurements of surface-wave phase could help to refine current elastic models of the Earth. 
In this paper, we develop further a two-station method to derive local amplification factors at seismic stations (Eddy and Ekström, 2011). We apply it to data recorded on the USArray and derive maps of local amplification across the footprint of the array. We assess the quality of calibration of USArray stations. Additionally, we make quantitative comparisons with predictions of local amplification made with mantle and crust models.

\section{Theory}

For a given angular frequency, $\omega$, surface-wave seismograms can be written as a function of amplitude and phase (e.g., Tromp and Dahlen, 1992, 1993);

$$
u(\omega)=A(\omega) \exp [i \Phi(\omega)],
$$

where $u(\omega)$ denotes the recorded displacement at the station, and $A(\omega)$ and $\Phi(\omega)$ are the amplitude and phase, respectively. The amplitude of the seismogram can be considered to be a product of four separate effects;

$$
A(\omega)=A_{S}(\omega) A_{R}(\omega) A_{F}(\omega) A_{Q}(\omega),
$$

where $A_{S}(\omega)$ is the effect of the source, $A_{R}(\omega)$ is the effect of the receiver, $A_{F}(\omega)$ is the effect of geometric spreading and focusing, and $A_{Q}(\omega)$ is the effect of attenuation (e.g., Dalton and Ekström, 2006b). Source effects include both earthquake source parameters and Earth structure local to the source. Receiver effects include both the instrument response and Earth structure local to the receiver. Because there are four contributions to each amplitude signal, there is inherent difficulty in isolating the effect of each. 
In this study, we form ratios of measured signal amplitudes recorded at adjacent stations to isolate the component of the surface-wave-amplitude signal local to the receiver. Consider the ratio of surface-wave amplitudes from one earthquake recorded at two stations denoted $i$ and $j$ (Note that although from here on we drop the frequency dependence in the equations, all quantities still depend on the frequency, $\omega$.$) ;$

$$
\frac{A_{i}}{A_{j}}=\frac{A_{S, i} A_{R, i} A_{F, i} A_{Q, i}}{A_{S, j} A_{R, j} A_{F, j} A_{Q, j}}
$$

For a given earthquake and station pair, all amplitude effects will contribute to the observed ratio. For nearby stations, the contribution to the ratio of the effects associated with the source and propagation is small, since the stations sample adjacent points of a coherent surface-wave wave field. Because takeoff angles and ray paths for waves recorded at two adjacent stations will be similar for a given earthquake, the ratios of all effects except the receiver can be expected to vary around 1.0. In contrast, local effects associated with receiver structure or instrument response will contribute a factor to the ratio that is consistent for all earthquakes recorded on the two stations.

To isolate the receiver effect, we first construct a datum, $d_{i j}^{k}$, from logarithmic amplitude ratios;

$$
d_{i j}^{k}=\ln \left(A_{i} / A_{j}\right)=\ln \left(A_{i}\right)-\ln \left(A_{j}\right),
$$

where $A_{i}$ and $A_{j}$ are the individual surface-wave amplitude measurements at a given frequency for each earthquake, $k$, that was recorded on two neighboring stations. For station pairs on which many earthquakes have been 
recorded, we then derive an average datum;

$$
\bar{d}_{i j}=\frac{1}{N_{E}} \sum_{k=1}^{N_{E}} d_{i j}^{k},
$$

where $N_{E}$ is the total number of earthquakes recorded on both stations in the pair.

We wish to attribute the average datum, $\bar{d}_{i j}$, to the difference between the local receiver effect at each station in the pair, $\ln \left(A_{R, i}\right)-\ln \left(A_{R, j}\right)$. The cancellation of source and path effects in the averaging should work best for stations that are separated by a small distance, for which the surface waves generated by a single earthquake have traveled along nearly identical paths. Ideally, the station separation is a fraction of the wavelength of the wave and of any local complexity in the wavefield.

In the surface-wave ray-theoretical framework, the receiver-amplitude factor, $A_{R}$, is a function of the local radial elastic structure and has two contributing factors (e.g., Tromp and Dahlen, 1992; Wang and Dahlen, 1994). First, the surface amplitudes of the displacement eigenfunction vary depending on the local structure. A second, typically smaller, effect is related to the local speed of propagation. Given a radial profile taken from a threedimensional Earth model, a predicted receiver-amplification factor can be calculated for a specific location;

$$
A_{R}=\frac{D}{D_{0}} \sqrt{\frac{U_{0}}{U}}
$$

where $D$ and $D_{0}$ are the displacement eigenfunctions for the receiver location in the three-dimensional model and a reference model, respectively, $U$ is the group velocity of the model, and $U_{0}$ is the group velocity of the reference 
model. As pointed out by Ferreira and Woodhouse (2007b), specific expressions for the receiver-amplification factor vary depending on the convention used in the normalization of the surface-wave eigenfunctions. Here we follow the normal-mode convention (e.g., Gilbert and Dziewoński, 1975; Ferreira and Woodhouse, 2007b).

\section{Data and Analysis}

The data used in this study are recordings on the USArray of earthquakes with $M_{W}>5.5$ occurring between January 2006 and December 2011. Amplitudes are measured for minor-arc arrivals of Rayleigh waves between periods of $25 \mathrm{~s}$ and $125 \mathrm{~s}$ from 2172 earthquakes recorded on a total of 1384 stations. Cumulative geographic station coverage through 2011 is over more than half of the continental United States.

Measurements of amplitude anomalies are made using the technique of Ekström et al. (1997). In this method, the model surface wave is first expressed as a function of amplitude and phase using the earthquake location and source geometry taken from the Global Centroid Moment Tensor (CMT) project (Dziewoński et al., 1981; Ekström et al., 2012) and using the excitation and propagation characteristics calculated for the Preliminary Reference Earth Model (PREM) (Dziewoński and Anderson, 1981). A misfit function, representing the difference between the modeled and observed waveforms, is then minimized in an iterative process in which phase and amplitude are varied to best fit the waveforms. The amplitude anomaly is the variation in amplitude relative to the spherical Earth synthetic prediction necessary to match the modeled waveform to the observation at each period. In prac- 
tice, this means that the amplitude ratios used in the analysis (equation 3) have been corrected for the small differences in source radiation pattern and propagation effects on a reference spherical Earth as well as for instrumentresponse functions.

For this analysis, we consider Rayleigh wave amplitudes measured on the vertical component. We select high-quality amplitude-anomaly measurements as our data. We only include measurements from earthquakes that are at a distance greater than $15^{\circ}$ away from the recording station. Using these single-station data, we form the logarithmic amplitude ratios for station pairs in the array and average the ratios for each pair as in equation 5.

A selection based on the maximum distance between stations in each pair is needed to ensure that the source, focusing, and attenuation effects will all be nearly equal for amplitudes of surface waves from one earthquake recorded at two different stations. The smaller the maximum distance between stations in each pair, the more likely it is that this assumption holds. At the same time, a small maximum distance between stations excludes a large portion of the raw amplitude dataset. A distance of two degrees was chosen as a compromise that minimizes the differences of source, focusing, and attenuation effects and retains a large amount of the data. For the USArray, this typically leads to about 20-30 station neighbors.

To suppress the source and path effects in the average logarithmic amplitude ratios, a sufficiently large number of recorded events must be included. After experimentation, we selected ten as the minimum number of earthquakes for the analysis. The station pairs that are excluded from our selection as a consequence of this criterion are primarily located on the eastern 
edge of the array, for which there is the least amount of data.

Table 1 provides information about the data used in this study. At long periods $(\geq 50 \mathrm{~s})$, significantly more data were used, reflecting the larger number of high-quality observations available. For each station-pair observation, $\bar{d}_{i j}$, we calculate an associated uncertainty, $\bar{\sigma}_{i j}$, by $\bar{\sigma}_{i j}=\sigma_{i j} / \sqrt{N_{E}}$, where $\sigma_{i j}$ is the standard deviation of the observations and $N_{E}$ is the total number of observations for each station pair. Since the observations are derived from multiple differences, the covariance will be significant for observations on station pairs which have one station in common, and $\bar{\sigma}_{i j}$ will not be a complete characterization of the data uncertainties. However, the $\bar{\sigma}_{i j}$ values provide a good relative measure of the uncertainties associated with different pairs. The mean of these station-pair uncertainties for each period is reported in Table 1 . The increasing uncertainty with decreasing period reflects the larger scatter in the short-period measurements. We find that the large scatter and smaller number of observations available at periods shorter than $35 \mathrm{~s}$ lead to unstable results. We therefore limit the further analysis to periods $35 \mathrm{~s}$ and longer to ensure that the path-dependent effects on surface-wave amplitudes are averaged out.

The average logarithmic amplitude ratio for each station pair is taken as the datum in a least-squares inversion for local station amplification factors by minimizing $\chi^{2}$ in the following equation;

$$
\chi^{2}=\sum_{i j} \frac{1}{\bar{\sigma}_{i j}^{2}}\left[\left(\ln \left(A_{R, i}\right)-\ln \left(A_{R, j}\right)\right)-\bar{d}_{i j}\right]^{2},
$$

where $A_{R, i}$ and $A_{R, j}$ are the station amplification factors that are inverted for and the datum, $\bar{d}_{i j}$, and uncertainty, $\bar{\sigma}_{i j}$, are defined as above. Only those stations that are linked together by observations are included in the 
inversion. Absolute amplification factors cannot be resolved because the data are derived from amplitude differences at each period, and we apply the constraint that the logarithmic station amplification factors must sum to a value of 0 across the array for each period using the method of Lagrange multipliers. With this constraint, the inverse problem is overdetermined and no additional regularization is necessary.

\section{Results}

Local amplification factors are derived by inversion for Rayleigh waves at discrete periods between $35 \mathrm{~s}$ and $125 \mathrm{~s}$. Figure 2 shows the range of local Rayleigh wave amplification variations at different periods. Local amplification factors are distributed around 1.0, with the spread of variations increasing with decreasing period. The observed variation in surface-wave amplitude due to effects local to the receiver reaches $\pm 10 \%$ at $125 \mathrm{~s}$ and exceeds $\pm 20 \%$ at $35 \mathrm{~s}$.

The average logarithmic amplitude ratios are explained well by the derived amplitude factors. Figure 3 shows the variance reduction of the local amplification factors at all periods considered in this study. Variance reduction is highest for long-period Rayleigh waves, reaching values up to $95 \%$. The variance reduction is slightly lower at short periods, which could be due to several different reasons, including the smaller number of high-quality measurements and higher variability in the short-period amplitudes due to focusing and deformation of the wavefront at a wavelength less than or on the order of the station spacing of the USArray $(70 \mathrm{~km})$.

To assess whether the local amplification factors explain the observations 
at the level of our estimated uncertainties, we calculate the goodness-of-fit parameter $\chi^{2} / M$ (Figure 3), where $M$ is the number of degrees of freedom, here the difference between the number of station-pair observations and the number of stations at each period. We find goodness-of-fit values in the range $0.5-0.7$. We attribute the apparent over-fitting (i.e., $\chi^{2} / M<1.0$ ) of the data to our incomplete consideration of covariance in the station-pair data.

Figure 4 shows maps of the station amplification factors for Rayleigh waves at periods of $35 \mathrm{~s}, 50 \mathrm{~s}, 75 \mathrm{~s}$, and $125 \mathrm{~s}$. The amplification factors are relative and can be considered to be local amplification (for values $>1.0$ ) or local deamplification (for values $<1.0$ ) of an incoming wavefield. The anomalies exhibit spatial correlation with topography and geologic structures. For example, at the shorter periods, very large amplification $(+15 \%)$ is seen in Colorado and in the Snake River Plain. Extreme deamplification (-15\%) is seen along the Gulf of Mexico.

A striking feature of the maps is the spatial coherence of anomalies at the level of a few percent. We investigate the length over which the anomalies are correlated by first calculating the absolute value of the differences in local amplification for pairs of stations at varying inter-station distances. For 0.5-degree bins, we then find the average absolute difference in amplification between stations in each distance range. Figure 5 shows the average amplification difference as a function of inter-station distance for Rayleigh wave amplification factors at the different periods. The distance over which the amplification factors are well correlated is short ( $<2$ degrees) at $35 \mathrm{~s}$ and increases at longer periods. Due to finite-frequency effects, this length reflects not only an average scale of structure, but also the averaging of amplification 
over a period-dependent length. The minimum difference, observed at short inter-station distances for all periods, can be considered a measure of the average quality of calibration of the stations. Based on this analysis, we infer that the relative calibration of USArray stations is very good, with average errors less than $2-4 \%$.

Because of the spatial coherence of anomalies, it is possible to identify outlier stations that have problems with instrument response and calibration. Many of these problem stations are visible by inspection of the amplificationfactor maps, often as values that are significantly lower than those for the stations surrounding them. One example is TA station N02C-TA, located at $40.8^{\circ} \mathrm{N}$ and $123.3^{\circ} \mathrm{W}$. This station has a factor of two difference in gain with respect to neighboring stations, and appears as a distinct negative anomaly in the otherwise smooth local Rayleigh wave amplification maps.

We define outliers at each period to be stations that have an amplification that is $>2 \sigma$ different from at least five neighboring stations, where $\sigma$ is the standard deviation of the observed amplification factors at that period (Figure 2). Here, neighboring stations are defined to be those stations that lie less than 1 degree away from the station of interest. Table 2 lists the outliers for local Rayleigh wave amplification determined using this criteria at different periods. At long periods, a larger number of stations are identified as outliers because there is less variability in the amplification. The anomalous amplification factors of outlier stations are isolated and do not affect the observed amplification at nearby stations. This indicates that we can also resolve variations in amplification that are due to structural anomalies local to each station. 


\section{Discussion}

Observed local Rayleigh wave amplification factors contain anomalies that are spatially coherent and show good correlation with topography and geologic structures (Figure 4). We attribute the local Rayleigh wave amplification factors to variations in local elastic structure beneath each station. We observe that the range in variation of the amplification factors (10-20\%, depending on the period) is always larger than our inferred estimate of the quality of calibration of USArray stations (Figure 5). For well-calibrated arrays, the effect of local structure on surface-wave amplitudes is larger than effects due to errors in instrument response.

Maps of local amplification factors show similarities to surface-wave phase velocity maps in the western United States (e.g., Lin and Ritzwoller, 2011). Locations of high amplification coincide with regions of extremely slow phase velocity in the Rocky Mountains and in the region of the Snake River Plain and Yellowstone Hotspot. Local amplification is also correlated with crustal thickness (Gilbert, 2012). Stations located in regions of thicker crust tend to have larger amplification factors, such as the Sierra Nevada Mountains, the Rocky Mountains in Colorado, and the Snake River Plain and Yellowstone Hotspot. A location with thinner crust, the Columbia Plateau, has lower amplification factors relative to surrounding stations. Even at long periods, there are clear spatial correlations with surface geologic features.

The sensitivity kernels in Figure 1 illustrate the difficulties of directly associating the amplitude anomalies with radial elastic structure. In contrast with phase-velocity kernels, which are dominantly positive, so that a slow $v_{P}$ or $v_{S}$ intrinsic anomaly at any depth results in a slow phase velocity, ampli- 
tude kernels change sign with depth. Thus, a shallow, slow $v_{S}$ anomaly will lead to deamplification while an anomaly located at greater depth will lead to an amplification. Additionally, at shallow depth the $v_{P}$ and $v_{S}$ sensitivities are of opposite sign. This effect may explain the extreme deamplification observed along the Gulf of Mexico, an area with a thick layer of low-velocity sediments with high $v_{P} / v_{S}$ (Laske and Masters, 1997; Bassin et al., 2000).

In order to investigate the extent to which our amplification observations are qualitatively and quantitatively consistent with existing knowledge of the elastic structure of the crust and mantle beneath North America, we make corresponding predictions based on two models. First, we predict surfacewave amplitudes at each station using a model consisting of PREM core and mantle (Dziewoński and Anderson, 1981) and crustal structure from CRUST2.0, a crustal model with $2^{\circ}$ by $2^{\circ}$ resolution (Bassin et al., 2000). For each station, a radial profile is taken from that location in the model and normal modes are calculated. The predicted vertical amplification factor is then calculated as in equation 6 , where $D$ denotes the vertical displacement eigenfunction for PREM and CRUST2.0 at that location and the reference model is PREM. Second, to assess the importance of mantle heterogeneity, we also predict surface-wave amplitudes at each station using the three-dimensional model ND08 (Nettles and Dziewoński, 2008), a radially anisotropic shear-velocity model of the mantle beneath North America, embedded in a lower resolution global model. The model incorporates crustal structure from CRUST2.0. Local amplification is predicted in the same manner using radial profiles from this model. The effect of density heterogeneity is included in the predictions of amplification; density in the crust is taken 
from CRUST2.0 and density in the mantle is taken from PREM.

Figure 6 shows the correlations between the observed amplification and local amplification predicted by these two models at periods between $35 \mathrm{~s}$ and $125 \mathrm{~s}$. At short periods ( $\leq 40 \mathrm{~s}$ ), predictions made by the two models are equally well correlated with observed amplification factors, reflecting the fact that they both incorporate and are dominated by the effects of CRUST2.0. At periods longer than $40 \mathrm{~s}$, elastic structure in the mantle begins to dominate the receiver-amplitude signal, leading to the large separation of the correlations between the observations and predictions from the two models. Correlations for predictions made by PREM with CRUST2.0 are low or slightly anticorrelated at long periods, indicating that lateral variations in crustal structure alone are not sufficient to predict accurate surface-wave amplitudes.

The correlation between observed amplification factors and local amplification predicted by ND08 is large at longer periods with a maximum at $100 \mathrm{~s}(R=0.64)$. Figure 7 shows maps of the local amplification predicted by model ND08 at periods of $35 \mathrm{~s}, 50 \mathrm{~s}, 75 \mathrm{~s}$, and $125 \mathrm{~s}$. The predicted range of amplification is $\pm 10 \%$ at $125 \mathrm{~s}$ and exceeds $\pm 30 \%$ at $35 \mathrm{~s}$. These predicted ranges agree well with the observed range in local amplification (Figure 2). Comparison between the predictions made by these two models demonstrates that models containing laterally varying mantle structure are necessary for accurate predictions of surface-wave amplitudes, despite the strong sensitivity of amplitudes to shallow crustal structure (Figure 1).

The agreement found between the observations and the predictions provides corroboration that our method of isolating the local amplification effect 
is working. We also find good agreement with the recent study of Lin et al. (2012b), who measured Rayleigh wave amplification using Helmholtz tomography. In their study, Lin et al. (2012b) corrected for the effects of focusing and defocusing by computing the curvature of the phase travel time. The visual agreement between our $50 \mathrm{~s}$ map of amplification and their $60 \mathrm{~s}$ map (the closest period available) is very good. At $60 \mathrm{~s}$, the correlation of the two sets of amplification factors is high $(R=0.67)$. The good agreement indicates that our observed amplification factors are not strongly biased by unmodeled elastic focusing effects, even though we do not explicitly correct for this effect on amplitudes. We believe that any existing bias due to focusing effects is largest at the shortest periods and negligible at the longest periods considered in this study.

There are clear differences between the amplification predicted by model ND08 and that observed from measurements of surface-wave amplitude, despite the good agreement at long periods. This suggests that surface-wave amplification factors can help constrain elastic models of the crust and upper mantle, potentially as an additional data set to invert for radial structure beneath the region in which they were measured. In particular, surface-wave amplification is more sensitive than phase velocity to structure in the crust (Figure 1), indicating that local amplification could improve constraints on shallow structure.

We have isolated and investigated local amplification separately from other effects on surface-wave amplitude, including both attenuation and elastic focusing. As a result, the derived local amplification factors could also be used as correction factors to constrain these path-dependent effects better. 
For example, correction of local amplification observations in an inversion for attenuation would reduce the risk that local elastic structure be erroneously mapped into anelastic structure.

\section{Conclusions}

We have developed a technique to derive local surface-wave amplification across an array of seismic stations and applied it to data recorded on the USArray. Observed Rayleigh wave amplification factors are spatially coherent and exhibit good correlation with topography and geologic features, indicating that they are strongly controlled by elastic structure local to each USArray station. Local amplification varies by $\pm 10 \%$ at a period of $125 \mathrm{~s}$, demonstrating that local elastic structure has a significant effect on observed surface-wave amplitudes even at long periods. Predictions of local amplification factors show a similar range to the observations but exhibit some differences in pattern, indicating that the surface-wave amplification factors can be used to refine current elastic models of the crust and upper mantle.

The quality of amplitude calibration of USArray stations is very good, and variations of surface-wave amplification due to the effect of local structure can be resolved at the level of a few percent. This local amplification effect should not be ignored when studying surface-wave amplitudes on well-calibrated seismic arrays. We are making local amplification factors derived in this study available online. 


\section{Acknowledgments}

We thank Michael Ritzwoller, Fred Pollitz, and a Guest Editor for their helpful comments to improve the manuscript. Spahr Webb provided many useful comments on an earlier version of this work. We also thank Fan-Chi Lin for providing his maps of local amplification for comparison. We are grateful to all involved in the deployment and operation of the USArray. The consistent and high quality data from the array made the research presented here possible. We also thank the IRIS DMC for providing access to these data. This research was supported by the EarthScope Program of the National Science Foundation, grant EAR-0952285.

Bassin, C., Laske, G., Masters, G., 2000. The Current Limits of Resolution for Surface Wave Tomography in North America. EOS Trans. AGU 81, F891.

Boschi, L., Ekström, G., 2002. New images of the Earth's upper mantle from measurements of surface wave phase velocity anomalies. J. Geophys. Res. $107,2059$.

Dalton, C.A., Ekström, G., 2006a. Constraints on global maps of phase velocity from surface-wave amplitudes. Geophys. J. Int. 167, 820-826.

Dalton, C.A., Ekström, G., 2006b. Global models of surface wave attenuation. J. Geophys. Res. 111, B05317.

Durek, J.J., Ritzwoller, M.H., Woodhouse, J.H., 1993. Constraining upper mantle anelasticity using surface wave amplitude anomalies. Geophys. J. Int. 114, 249-272. 
Dziewoński, A.M., Anderson, D.L., 1981. Preliminary Reference Earth Model. Phys. Earth Planet. In. 25, 297-356.

Dziewoński, A.M., Chou, T.A., Woodhouse, J.H., 1981. Determination of earthquake source parameters from waveform data for studies of global and regional seismicity. J. Geophys. Res. 86, 2825-2852.

Eddy, C., Ekström, G., 2011. Surface Wave Amplitude Anomalies in the Western United States. Abstract S41A-2153 presented at 2011 Fall Meeting, AGU, San Francisco, Calif., 5-9 Dec. .

Ekström, G., 2011. A global model of Love and Rayleigh surface wave dispersion and anisotropy, 25-250 s. Geophys. J. Int. 187, 1668-1686.

Ekström, G., Dalton, C.A., Nettles, M., 2006. Observations of Timedependent Errors in Long-period Instrument Gain at Global Seismic Stations. Seismol. Res. Lett. 77, 12-22.

Ekström, G., Nettles, M., Dziewoński, A.M., 2012. The global CMT project 2004-2010: Centroid-moment tensors for 13,017 earthquakes. Phys. Earth Planet. In. 200-201, 1-9.

Ekström, G., Tromp, J., Larson, E.W.F., 1997. Measurements and global models of surface wave propagation. J. Geophys. Res. 102, 8137-8157.

Ferreira, A.M.G., Woodhouse, J.H., 2007a. Observations of long period Rayleigh wave ellipticity. Geophys. J. Int. 169, 161-169.

Ferreira, A.M.G., Woodhouse, J.H., 2007b. Source, path and receiver effects on seismic surface waves. Geophys. J. Int. 168, 109-132. 
Gilbert, F., Dziewoński, A.M., 1975. An Application of Normal Mode Theory to the Retrieval of Structural Parameters and Source Mechanisms from Seismic Spectra. Philos. T. Roy. Soc. A 278, 197-269.

Gilbert, H., 2012. Crustal structure and signatures of recent tectonism as influenced by ancient terranes in the western United States. Geosphere 8, $141-157$.

Gung, Y., Romanowicz, B., 2004. Q tomography of the upper mantle using three-component long-period waveforms. Geophys. J. Int. 157, 813-830.

Kustowski, B., Ekström, G., Dziewoński, A.M., 2008. Anisotropic shear-wave velocity structure of the Earth's mantle: A global model. J. Geophys. Res. 113, B06306.

Laske, G., Masters, G., 1997. A Global Digital Map of Sediment Thickness. EOS Trans. AGU 78, F483.

Lin, F.C., Ritzwoller, M.H., 2011. Helmholtz surface wave tomography for isotropic and azimuthally anisotropic structure. Geophys. J. Int. 186, 11041120.

Lin, F.C., Schmandt, B., Tsai, V.C., 2012a. Joint inversion of Rayleigh wave phase velocity and ellipticity using USArray: Constraining velocity and density structure in the upper crust. Geophys. Res. Lett. 39, L12303.

Lin, F.C., Tsai, V.C., Ritzwoller, M.H., 2012b. The local amplification of surface waves: A new observable to constrain elastic velocities, density, and anelastic attenuation. J. Geophys. Res. 117, B06302. 
Masters, G., Johnson, S., Laske, G., Bolton, H., Davies, J.H., 1996. A ShearVelocity Model of the Mantle [and Discussion]. Philos. T. Roy. Soc. A 354, $1385-1411$.

Nettles, M., Dziewoński, A.M., 2008. Radially anisotropic shear velocity structure of the upper mantle globally and beneath North America. J. Geophys. Res. 113, B02303.

Pollitz, F.F., Snoke, J.A., 2010. Rayleigh-wave phase-velocity maps and three-dimensional shear velocity structure of the western US from local non-plane surface wave tomography. Geophys. J. Int. 180, 1153-1169.

Ritsema, J., Deuss, A., van Heijst, H.J., Woodhouse, J.H., 2011. S40RTS: a degree-40 shear-velocity model for the mantle from new Rayleigh wave dispersion, teleseismic traveltime and normal-mode splitting function measurements. Geophys. J. Int. 184, 1223-1236.

Selby, N.D., Woodhouse, J.H., 2000. Controls on Rayleigh wave amplitudes: attenuation and focusing. Geophys. J. Int. 142, 933-940.

Selby, N.D., Woodhouse, J.H., 2002. The Q structure of the upper mantle: Constraints from Rayleigh wave amplitudes. J. Geophys. Res. 107, 2097.

Shapiro, N.M., Ritzwoller, M.H., 2002. Monte-Carlo inversion for a global shear-velocity model of the crust and upper mantle. Geophys. J. Int. 151, 88-105.

Tanimoto, T., Rivera, L., 2008. The ZH ratio method for long-period seismic data: sensitivity kernels and observational techniques. Geophys. J. Int. $172,187-198$. 
Trampert, J., Woodhouse, J.H., 1995. Global phase velocity maps of Love and Rayleigh waves between 40 and 150 seconds. Geophys. J. Int. 122, 675-690.

Trampert, J., Woodhouse, J.H., 2003. Global anisotropic phase velocity maps for fundamental mode surface waves between 40 and 150 s. Geophys. J. Int. 154, 154-165.

Tromp, J., Dahlen, F.A., 1992. Variational principles for surface wave propagation on a laterally heterogeneous Earth-II. Frequency-domain JWKB theory. Geophys. J. Int. 109, 599-619.

Tromp, J., Dahlen, F.A., 1993. Variational principles for surface wave propagation on a laterally heterogeneous Earth-III. Potential representation. Geophys. J. Int. 112, 195-209.

Wang, Z., Dahlen, F.A., 1994. JWKB surface-wave seismograms on a laterally heterogeneous earth. Geophys. J. Int. 119, 381-401.

Yang, Y., Forsyth, D.W., 2006. Rayleigh wave phase velocities, small-scale convection, and azimuthal anisotropy beneath southern California. J. Geophys. Res. 111, B07306.

Yano, T., Tanimoto, T., Rivera, L., 2009. The ZH ratio method for longperiod seismic data: inversion for S-wave velocity structure. Geophys. J. Int. $179,413-424$.

Zhang, Y.S., Tanimoto, T., 1991. Global Love wave phase velocity variation and its significance to plate tectonics. Phys. Earth Planet. In. 66, 160-202. 


\begin{tabular}{ccccc}
\hline Period (sec) & $N_{S}$ & $N_{P}$ & $\bar{N}_{E}$ & $\overline{\bar{\sigma}}$ \\
\hline 25 & 1065 & 12930 & 20.46 & 0.076 \\
30 & 1065 & 12930 & 20.46 & 0.061 \\
35 & 1232 & 17080 & 34.00 & 0.047 \\
40 & 1232 & 17080 & 34.00 & 0.040 \\
45 & 1232 & 17080 & 34.00 & 0.035 \\
50 & 1303 & 18956 & 58.07 & 0.030 \\
75 & 1303 & 18956 & 58.07 & 0.015 \\
100 & 1303 & 18956 & 58.07 & 0.011 \\
125 & 1300 & 18911 & 57.52 & 0.013 \\
\hline
\end{tabular}

Table 1: Data used in this study. Number of stations, $N_{S}$, number of station pairs, $N_{P}$, average number of observations per station pair, $\bar{N}_{E}$, and average station-pair uncertainty, $\overline{\bar{\sigma}}$. 


\begin{tabular}{ccccccc}
\hline Station & Latitude & Longitude & $35 \mathrm{~s}$ & $50 \mathrm{~s}$ & $75 \mathrm{~s}$ & $125 \mathrm{~s}$ \\
\hline $343 \mathrm{~A}-\mathrm{TA}$ & $31.284^{\circ} \mathrm{N}$ & $91.617^{\circ} \mathrm{W}$ & - & 0.77 & 0.78 & $\mathbf{1 . 0 9}$ \\
$\mathrm{H} 17 \mathrm{~A}-\mathrm{TA}$ & $44.395^{\circ} \mathrm{N}$ & $110.576^{\circ} \mathrm{W}$ & - & 1.24 & $\mathbf{1 . 5 1}$ & 1.11 \\
IBP-CI & $32.661^{\circ} \mathrm{N}$ & $116.093^{\circ} \mathrm{W}$ & 1.01 & 1.08 & 1.17 & $\mathbf{1 . 2 0}$ \\
ISA-CI & $35.663^{\circ} \mathrm{N}$ & $118.474^{\circ} \mathrm{W}$ & 0.86 & $\mathbf{0 . 7 7}$ & $\mathbf{0 . 7 2}$ & $\mathbf{0 . 6 3}$ \\
$\mathrm{J} 17 \mathrm{~A}-\mathrm{TA}$ & $43.363^{\circ} \mathrm{N}$ & $110.712^{\circ} \mathrm{W}$ & $\mathbf{0 . 8 7}$ & $\mathbf{0 . 8 5}$ & $\mathbf{0 . 8 4}$ & $\mathbf{0 . 8 0}$ \\
$\mathrm{MSO}-\mathrm{US}$ & $46.829^{\circ} \mathrm{N}$ & $113.941^{\circ} \mathrm{W}$ & $\mathbf{0 . 7 0}$ & $\mathbf{0 . 6 7}$ & $\mathbf{0 . 6 7}$ & $\mathbf{0 . 6 5}$ \\
$\mathrm{N} 02 \mathrm{C}-\mathrm{TA}$ & $40.822^{\circ} \mathrm{N}$ & $123.306^{\circ} \mathrm{W}$ & $\mathbf{0 . 6 2}$ & $\mathbf{0 . 5 7}$ & $\mathbf{0 . 5 7}$ & $\mathbf{0 . 5 5}$ \\
$\mathrm{OSI}-\mathrm{CI}$ & $34.614^{\circ} \mathrm{N}$ & $118.724^{\circ} \mathrm{W}$ & 0.95 & 0.90 & 0.91 & $\mathbf{0 . 8 7}$ \\
$\mathrm{S} 43 \mathrm{~A}-\mathrm{TA}$ & $37.572^{\circ} \mathrm{N}$ & $90.075^{\circ} \mathrm{W}$ & $\mathbf{0 . 5 4}$ & $\mathbf{0 . 5 3}$ & $\mathbf{0 . 4 7}$ & $\mathbf{0 . 5 0}$ \\
$\mathrm{SAO}-\mathrm{BK}$ & $36.764^{\circ} \mathrm{N}$ & $121.447^{\circ} \mathrm{W}$ & 0.82 & 0.89 & 0.91 & $\mathbf{0 . 9 0}$ \\
$\mathrm{T} 41 \mathrm{~A}-\mathrm{TA}$ & $37.044^{\circ} \mathrm{N}$ & $91.764^{\circ} \mathrm{W}$ & 0.86 & 0.93 & 0.83 & $\mathbf{0 . 8 0}$ \\
$\mathrm{X} 41 \mathrm{~A}-\mathrm{TA}$ & $34.495^{\circ} \mathrm{N}$ & $92.514^{\circ} \mathrm{W}$ & 0.97 & $\mathbf{1 . 1 7}$ & $\mathbf{1 . 1 0}$ & $\mathbf{0 . 8 6}$ \\
$\mathrm{YBH}-\mathrm{BK}$ & $41.732^{\circ} \mathrm{N}$ & $122.710^{\circ} \mathrm{W}$ & 1.12 & 1.04 & 0.94 & $\mathbf{0 . 8 3}$ \\
\hline
\end{tabular}

Table 2: Outlier stations, latitudes, longitudes, and local amplification factors found for Rayleigh waves at periods of $35 \mathrm{~s}, 50 \mathrm{~s}, 75 \mathrm{~s}$, and $125 \mathrm{~s}$. Bold entries are amplification factors which are $>2 \sigma$ from the values of at least 5 neighboring stations, where $\sigma$ is the standard deviation of the amplification factors at each period. Blank entries indicate that the station was not included in the inversion at that period. 

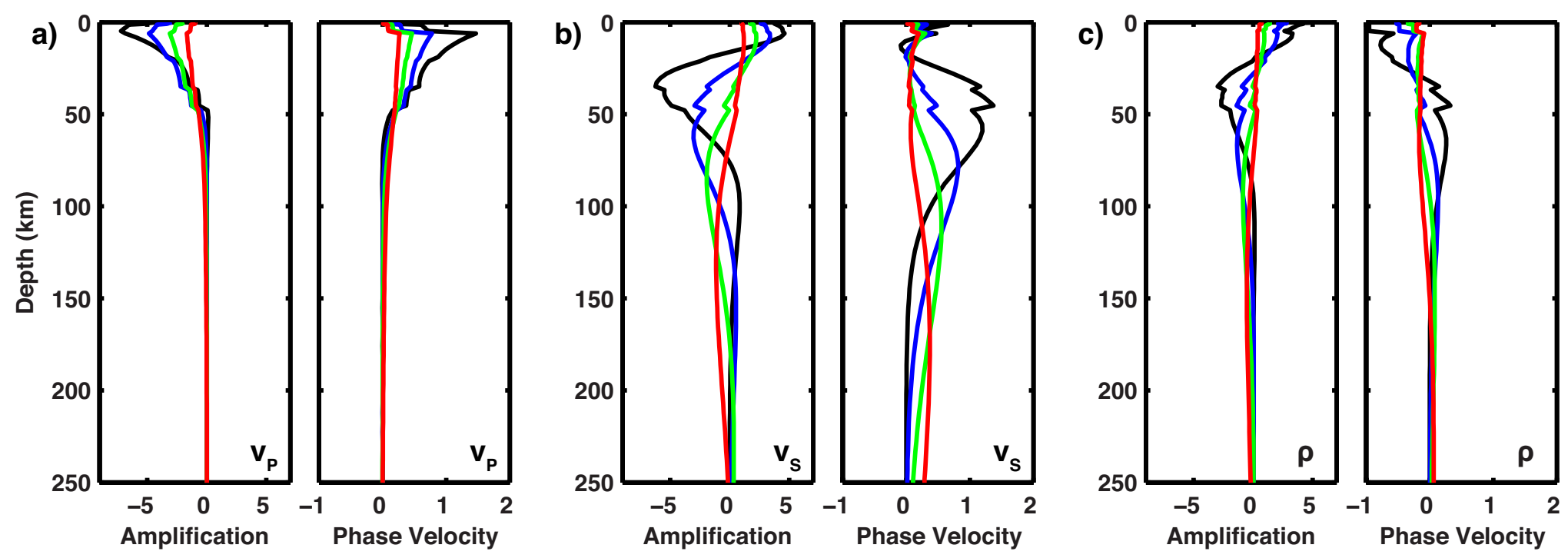

Figure 1: a) $v_{P}$, b) $v_{S}$, and c) density, $\rho$, sensitivity kernels for Rayleigh wave amplification (left) and phase velocity (right) at periods of $35 \mathrm{~s}$ (black), $50 \mathrm{~s}$ (blue), $75 \mathrm{~s}$ (green), and 125 s (red), calculated using a one-dimensional profile from ND08 (Nettles and Dziewoński, 2008) and CRUST2.0 (Bassin et al., 2000) located at $35^{\circ} \mathrm{N}$ and $99^{\circ} \mathrm{W}$. Sensitivity kernels are the relative amplitude or phase velocity perturbation due to a $1 \%$ velocity or density increase in a $1 \mathrm{~km}$ thick layer. Note that the units of amplification and phase velocity are $\times 10^{-5} \%$ and the amplification and phase velocity kernels are always plotted on the same scale. 


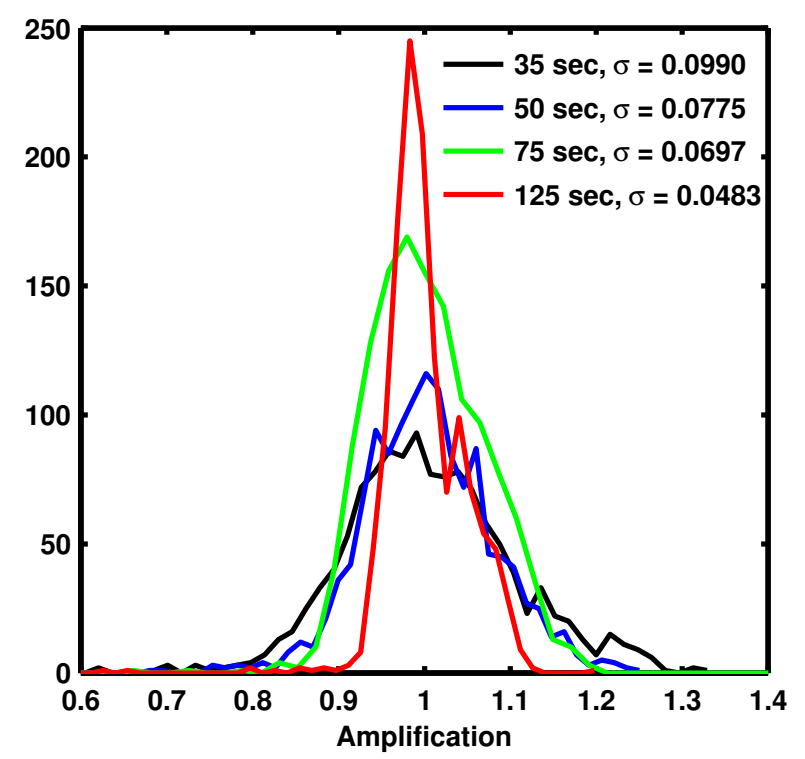

Figure 2: Histogram showing the distribution of local Rayleigh wave amplification factors at periods of $35 \mathrm{~s}, 50 \mathrm{~s}, 75 \mathrm{~s}$, and $125 \mathrm{~s}$ and the standard deviation, $\sigma$, at each period. Observed variations in local amplification reach $\pm 10 \%$ at $125 \mathrm{~s}$. The range of variation in local amplification is larger at short periods. 

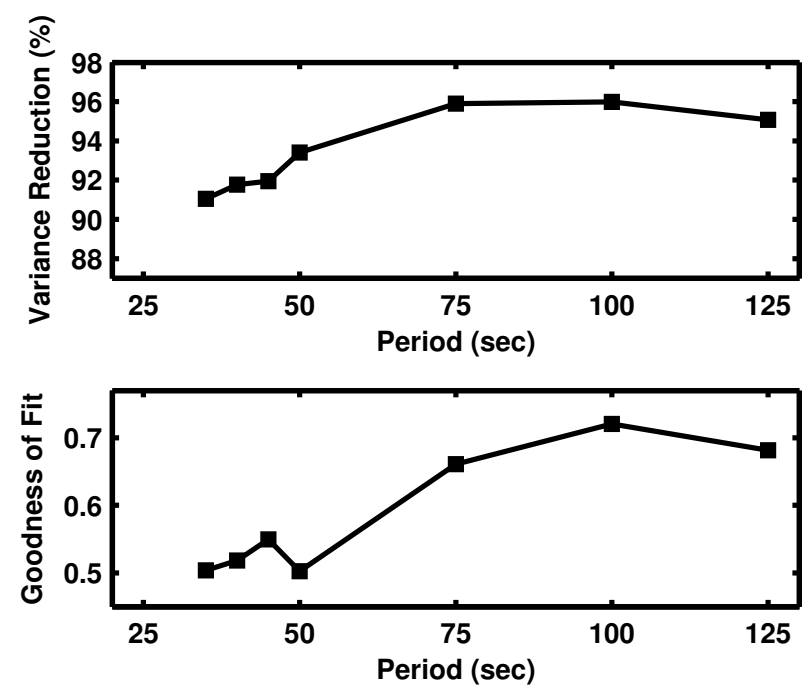

Figure 3: Variance reduction (top) and goodness-of-fit (bottom) for Rayleigh wave amplification factors at periods between $35 \mathrm{~s}$ and $125 \mathrm{~s}$. Variance reduction is high for all periods. The goodness-of-fit parameter is equal to $\chi^{2} / M$, where $M$ is the number of degrees of freedom. We attribute over-fitting (i.e., $\chi^{2} / M<1.0$ ) of the data to overestimated uncertainties. 

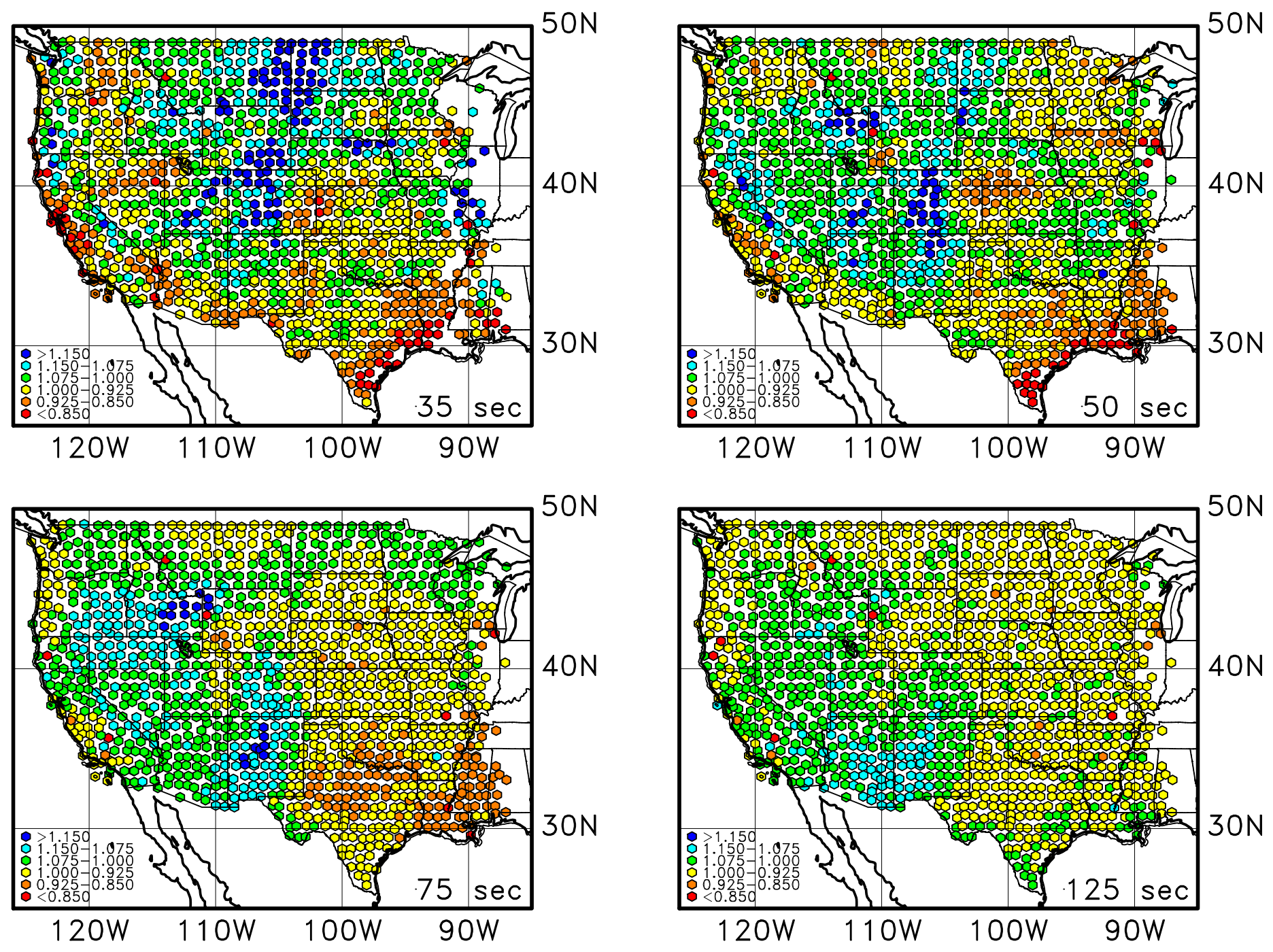

Figure 4: Observed local Rayleigh wave amplification factors at periods of 35 s, 50 s, 75 $\mathrm{s}$, and $125 \mathrm{~s}$. Each symbol corresponds to one USArray station and the color represents the derived amplification factor. 


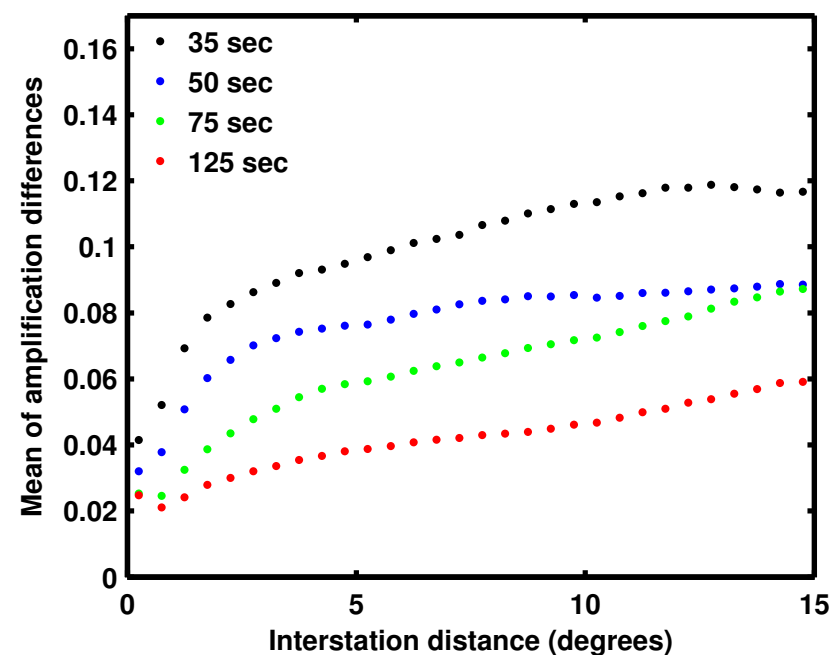

Figure 5: Average absolute differences between local Rayleigh wave amplification factors at stations as a function of inter-station distance at periods of $35 \mathrm{~s}, 50 \mathrm{~s}, 75 \mathrm{~s}$, and 125 s. Observed amplification factors are best correlated at short inter-station distances. The minimum difference $(2-4 \%)$ is an estimate of the average quality of calibration of the stations in the array. 


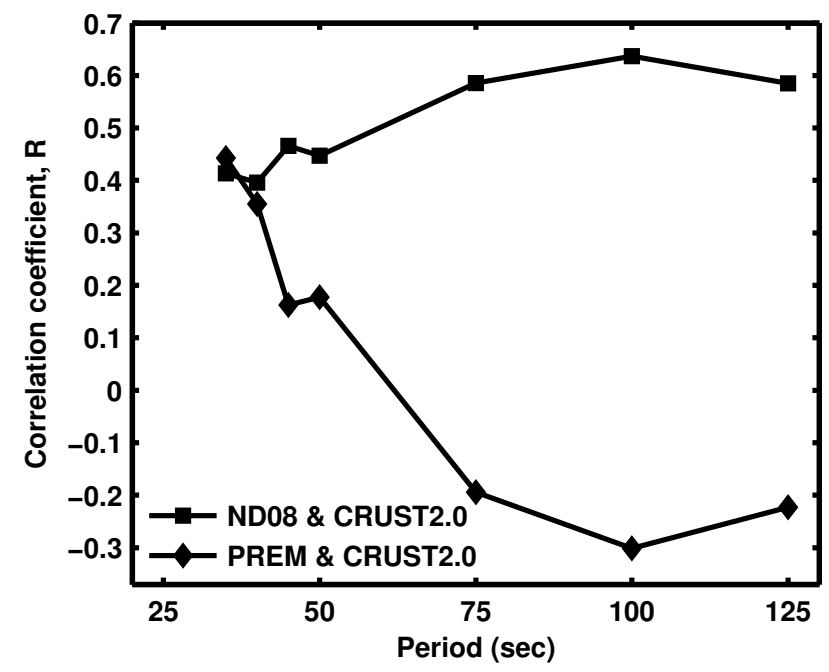

Figure 6: Correlation coefficients, $R$, between observed local Rayleigh wave amplification factors and predictions of local amplification factors made using models ND08 with CRUST2.0 and PREM with CRUST2.0 at periods between $35 \mathrm{~s}$ and $125 \mathrm{~s}$. Predictions made at long periods from model ND08 are best correlated with observed amplification factors. 

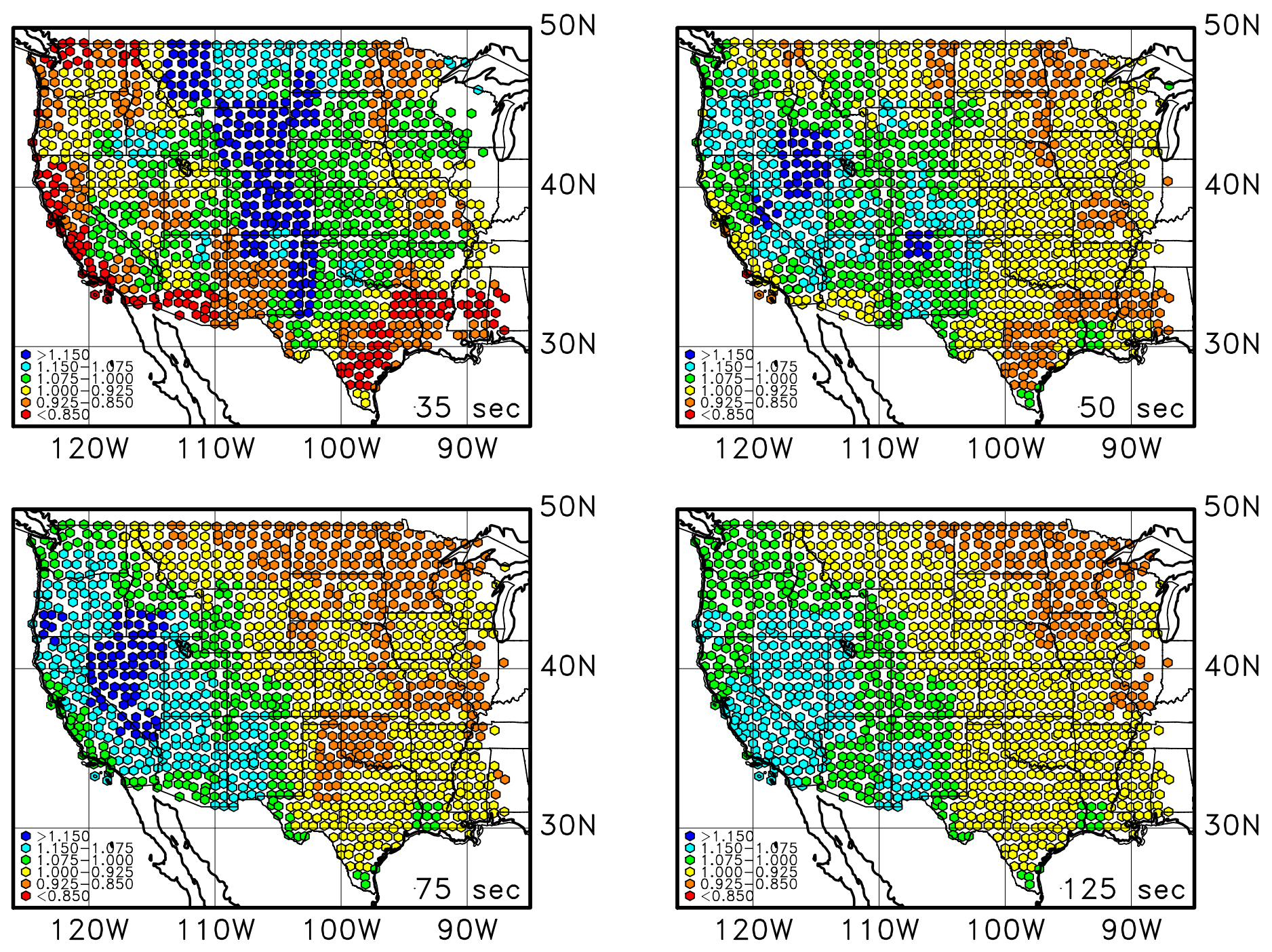

Figure 7: Predicted local Rayleigh wave amplification factors at periods of $35 \mathrm{~s}, 50 \mathrm{~s}, 75$ s, and 125 s made using model ND08 with CRUST2.0. Each symbol corresponds to one USArray station and the color represents the predicted local amplification factor. Amplification factors are normalized to the mean value at each period for better comparison with the maps of observed amplification and the color scale is the same as in Figure 4. 